\title{
Image potential states at chevron-shaped graphene nanoribbons /Au(111) interfaces
}

\author{
Christopher Bronner, ${ }^{1,2, *}$ Anton Haase, ${ }^{2}$ and Petra Tegeder ${ }^{1}$ \\ ${ }^{1}$ Ruprecht-Karls-Universität Heidelberg, Physikalisch-Chemisches Institut, Im Neuenheimer Feld 253, 69120 Heidelberg, Germany \\ ${ }^{2}$ Freie Universität Berlin, Fachbereich Physik, Arnimallee 14, 14195 Berlin, Germany
}

(Received 1 December 2014; revised manuscript received 7 January 2015; published 23 January 2015)

\begin{abstract}
Image potential states (IPSs) have been observed for various adsorbed carbon structures, such as graphene or carbon nanotubes. Graphene nanoribbons (GNRs) are intriguing nanostructures with a significant band gap which promise applications in nanotechnology. In the present paper we employ two-photon photoemission (2PPE) to investigate the unoccupied electronic structure and particularly the IPS of chevron-shaped GNR which are synthesized in a thermally activated on-surface synthesis on $\mathrm{Au}(111)$. Angle- and time-resolved 2PPE are utilized to gain further insights into the properties of the IPS. Compared to the pristine surface, reduced effective masses between 0.6 and 0.8 electron masses are observed and the lifetimes of the IPS are below the experimental detection limit, which is in the femtosecond regime. Independent of the concentration of $\mathrm{N}$ dopant atoms introduced in the GNR we observe a constant binding energy with respect to the vacuum level of the system.
\end{abstract}

DOI: 10.1103/PhysRevB.91.045428

PACS number(s): 73.20.At, 73.22.Pr

\section{INTRODUCTION}

Despite the intriguing electronic properties of graphene [1-3], there are limitations to this two-dimensional material which need to be overcome, such as the fact that it does not possess a finite band gap. In order to address this problem, there are various approaches such as employing mechanical strain [4-6], using biased bilayers of graphene [7,8], or using lateral confinement of charge carriers in quasi-onedimensional graphene nanoribbons (GNRs) [9,10].

The electronic structure of GNRs, and particularly their one-dimensional band structure, can be manipulated by altering key properties of the GNRs such as the overall width [11-13], the chirality $[9,10]$, or the concentration of dopant atoms [14-16]. GNR fabrication using lithographic patterning of graphene sheets $[11,17]$ or unzipping of carbon nanotubes $[18,19]$, which yields relatively wide ribbons with high defect concentrations, seems unsuitable for manufacturing GNRs for device applications. The reason is the high sensitivity of the electronic structure and the need to produce narrow GNRs on the nanometer scale in order to reach technologically relevant band gaps on the scale of a few electron-volts.

Unlike these top-down techniques, the bottom-up synthesis based on molecular precursors allows for the fabrication of well-defined GNRs. This is accomplished by deposition of halogen-substituted precursor molecules on noble metal surfaces [typically $\mathrm{Au}(111)$ ], thermal dissociation of the halogen, and subsequent covalent coupling of the molecules. In a second step at higher temperatures, the so-formed adsorbed polymer undergoes a cyclodehydrogenation reaction which produces the fully aromatic GNR [20,21].

Image potential states (IPSs) are a prominent feature of a variety of clean or adsorbate-covered metal surfaces. They arise due to the screening of an electron on the vacuum side of the surface and the resulting attractive interaction between this electron and its image charge [22-26]. The corresponding potential depends inversely on the surface-electron distance and is therefore similar to the radial potential of a hydrogen

*bronner@zedat.fu-berlin.de atom. The eigenstates of the Schrödinger equation of this system are thus a series of Rydberg-like states which converge to the vacuum energy. Unlike substrate bands or molecular orbitals of an adsorbate, these IPSs are therefore pinned to the vacuum level rather than the Fermi level. Assuming a negligible quantum defect and thus approximating the substrate as an infinitely large potential wall, the first IPS has a binding energy of $0.85 \mathrm{eV}$ with respect to the vacuum energy. While IPSs are localized in the direction of the surface normal, they are delocalized parallel to the surface and thus have a two-dimensional nature with a quasi-free electron dispersion. Due to the overlap of the IPS wave function with substrate bands, the IPSs possess a small lifetime $\tau$, which increases with increasing quantum number $n$ as $\tau \propto n^{3}$ [22]. These unoccupied electronic states which are localized at the sample surface and possess lifetimes on the femtosecond time scale are ideally suited for investigation with time-resolved two-photon photoemission (2PPE) [22,27].

For the clean $\mathrm{Au}(111)$ surface, the first $(n=1)$ IPS has been observed at binding energies between 0.60 and $0.42 \mathrm{eV}$ with respect to the vacuum level and with an effective mass $m_{\text {eff }}$ of $1.0 \pm 0.2 m_{\mathrm{e}}[28,29]$.

IPSs have been observed for graphene-covered surfaces using scanning tunneling spectroscopy $[30,31]$ as well as 2PPE [32-35]. A comparative study using various underlying metal substrates demonstrates that the influence of the substrate is minor and that the screening is mostly a property of the adsorbed graphene layer [36,37]. Besides extended graphene layers, IPSs have also been observed for adsorbed nanographenes [38] and carbon nanotubes [39,40]. For the above-mentioned, flat GNR, however, IPSs have not been investigated so far (during review of the present paper, another 2PPE study of excited states of GNR on $\mathrm{Au}(788)$ was published [41]).

Using 2PPE, we studied the IPS of a chevron-shaped GNR (CGNR; see Fig. 1)/Au(111) interface [20,42,43]. We find a binding energy of $0.91 \mathrm{eV}$ with respect to the vacuum level of the sample, an effective mass of $0.63 m_{\mathrm{e}}$, and a lifetime below the temporal resolution of our setup (62 fs). By comparison with two other CGNRs with different $\mathrm{N}$ doping concentrations, we can follow the IPS pinning to the vacuum level. 


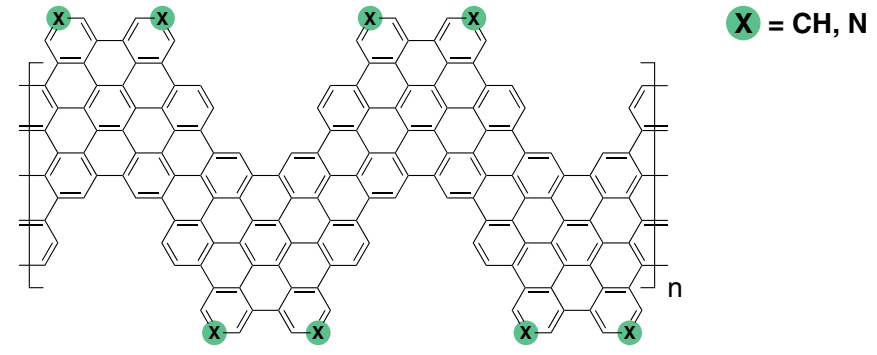

FIG. 1. (Color online) Chevron-shaped GNR. While the unsubstituted CGNR does not contain any nitrogen $(\mathrm{N})$, the singly (doubly) doped precursors lead to CGNR with half (all) of the highlighted dopant sites substituted with $\mathrm{N}$.

\section{EXPERIMENTAL METHODS}

The CGNRs were fabricated via thermally activated onsurface synthesis as described originally in Ref. [20]. The (111) surface of a Au single crystal was prepared by $\mathrm{Ar}^{+}$ sputtering ( $1.5 \mathrm{keV}$ ion energy) and annealing at $800 \mathrm{~K}$. The molecular precursor 6,11-dibromo-1,2,3,4-tetraphenyltriphenylene, which was synthesized in the group of Stefan Hecht (Humboldt-Universität zu Berlin), was evaporated from a Knudsen cell at $410 \mathrm{~K}$ and deposited onto the cold surface (held at $120 \mathrm{~K}$ ) in ultrahigh vacuum. Doped CGNRs were produced by evaporation of singly and doubly $\mathrm{N}$-doped precursor molecules (evaporation temperatures were $470 \mathrm{~K}$ and $450 \mathrm{~K}$, respectively), as described in Ref. [43]. The bottomup synthesis reaction was initiated by annealing the precursorcovered surface at $710 \mathrm{~K}$ for $10 \mathrm{~min}$. Subsequent $2 \mathrm{PPE}$ measurements were carried out at cryostatic temperatures below $100 \mathrm{~K}$. While our methods do not allow to determine the length of the produced GNR, scanning tunneling microscopy measurements have previously shown that the typical length is on the order of tens of nanometers [20].

Characterization of the undoped and the doped CGNRs as well as the intermediate reaction products was done using high-resolution electron energy loss spectroscopy (HREELS), as described in Ref. [43]. This method provides information on the adsorbate geometry during the different stages of the on-surface synthesis reaction by means of vibrational spectroscopy. For example, the final reaction product (the CGNR) exhibits a reduced number of vibrational modes and clearly possesses a flat adsorption geometry of the aromatic system.

Since the HREELS experiments were carried out in a different setup, an additional characterization in our own setup was done using temperature-programmed desorption (TPD; see Fig. 2). There the desorption of different molecular fragments was monitored using a quadrupole mass spectrometer (QMS) while the temperature was increased with a constant heating rate $\beta$. The desorption features in Fig. 2 are in analogy to those observed during the on-surface synthesis of a similar bottom-up fabricated GNR [44]. At a sample temperature of around $400 \mathrm{~K}$, desorption features due to fragments of the molecular precursor (91 amu, $\mathrm{C}_{7} \mathrm{H}_{7}^{+}$and $158 \mathrm{amu}, \mathrm{C}_{6} \mathrm{H}_{5} \mathrm{Br}$ ) indicate desorption of the second and higher-lying adsorbate layers. Note that the fragment at $158 \mathrm{amu}$ does not suggest desorption of ${ }^{79} \mathrm{Br}_{2}$ since this should also be reflected in a

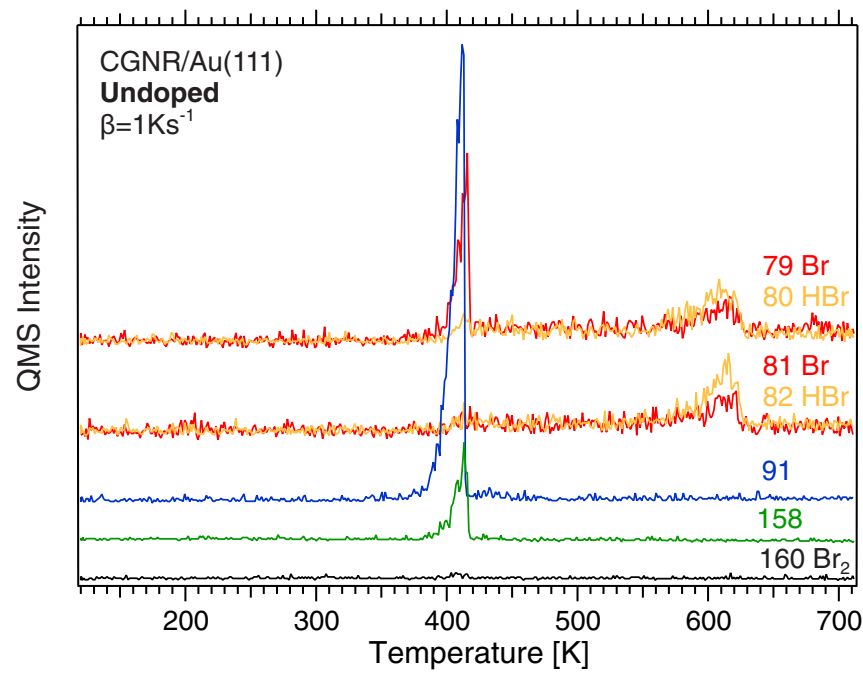

FIG. 2. (Color online) TPD curves recorded during the onsurface synthesis of the undoped CGNR. The heating rate was $\beta=1 \mathrm{~K} \mathrm{~s}^{-1}$. The measured fragment masses (in $\mathrm{amu}$ ) are stated next to the respective curves in the figure. A linear background was subtracted from all TPD curves.

desorption feature of ${ }^{81} \mathrm{Br}^{79} \mathrm{Br}$ at $160 \mathrm{amu}$, which is not the case. At around $550 \mathrm{~K}$, desorption features are observed for $\mathrm{H}^{79} \mathrm{Br}$ and $\mathrm{H}^{81} \mathrm{Br}$, as well as for their respective fragments consisting of only one $\mathrm{Br}$ atom. In full analogy to the system described in Ref. [44], this associative desorption of $\mathrm{HBr}$ is a side-effect of the final cyclodehydrogenation step of the on-surface synthesis reaction. Note that the desorption peak of the 79 -amu trace at around $400 \mathrm{~K}$ is due to the doubly ionized fragment of the molecular precursor with a mass of $158 \mathrm{amu}$. TPD of the doped species resulted in a similar desorption behavior.

The IPS of the CGNR were studied with 2PPE, where a pump laser pulse with photon energy $h v_{1}$ induces electronic transitions from occupied electronic states of the substrate or the adsorbate into unoccupied electronic states. These excited states are then probed with a second laser pulse with photon energy $h v_{2}$ by photoemission of the excited electron. Being a photoemission technique, 2PPE is surface-sensitive and therefore particularly suited to study adsorbate systems, as well as IPSs, which are localized at the surface [23,45-47]. Since features of the photoelectron spectrum may arise from occupied (excited via a virtual intermediate state) or unoccupied states the spectra are shown over the final-state energy of the photoemitted electron with respect to the Fermi level $E_{\mathrm{F}}, E_{\text {Final }}-E_{\mathrm{F}}=\Phi+E_{\text {kin }}$, where $\Phi$ is the global work function and $E_{\mathrm{kin}}$ is the kinetic energy of the photoelectron.

In analogy to conventional photoemission of occupied states, detection of photoemitted electrons at different emission angles (angle-resolved 2PPE, AR-2PPE) makes it possible to study the dispersion of electronic states parallel to the surface since the electron momentum parallel to the surface $k_{\|}$is conserved in the photoemission process. The pump-probe scheme furthermore enables us to study the population dynamics of excited electronic states on the time scale of the femtosecond laser pulses by delaying the probe pulse with respect to the pump pulse (time-resolved 2PPE, TR-2PPE). 
In the most simple case of a single de-excitation channel, the time-dependent population $N(t)=\exp (-t / \tau)$ leads to a broadening of the 2PPE intensity as a function of pumpprobe delay, the so-called cross-correlation trace which is a convolution of $N(t)$ with the temporal profiles of the two laser pulses.

The laser pulses are created in a Ti:sapphire oscillator and amplified in a regenerative amplifier. An optical parametric amplifier then provides tunable laser pulses in the visible spectrum which can be frequency doubled with a $\beta$ barium borate crystal, yielding tunable UV pulses. The laser beam is incident on the sample surface at an angle of $45^{\circ}$ and $p$ polarized. The photoelectrons are detected using a time-of-flight spectrometer in normal emission, unless otherwise stated. Angle-resolved measurements are performed by rotating the sample in front of the spectrometer. A detailed description of the experimental setup can be found elsewhere [48-50].

\section{RESULTS}

In the following we discuss the results of the 2PPE measurements conducted on the undoped CGNR. We then compare the results to those of the respective measurements of the two doped systems. The detailed discussion of the corresponding experiments can be found in the Supplemental Material [52].

Figure 3(a) shows a series of one-color 2PPE (1C-2PPE, pump and probe have the same photon energy $h v$ ) spectra for various photon energies $h v$. The global work function $\Phi$ can be determined from the low-energy cutoff edge of the 2PPE spectra and amounts to $4.66 \pm 0.02 \mathrm{eV}$, which is significantly lower than the work function of the pristine $\mathrm{Au}(111)$ surface which lies between 5.3 and $5.6 \mathrm{eV}$ [51,53]. In the final-state energy range up to around $7 \mathrm{eV}$, only contributions from the $d$-bands of the gold substrate are observed which are a common feature in 2PPE [48,54].

Close to the Fermi cutoff edge at higher energies, two additional features are found which shift as the photon energy changes, indicating that these features do not represent final states [55]. At low $h v$, those peaks cannot be observed in the spectrum anymore, which demonstrates that the corresponding electronic states cannot be occupied because then the energetic distance to the Fermi edge would be independent of the photon energy. We may thus conclude that these states arise due to unoccupied electronic states at energies of $3.75 \pm 0.07 \mathrm{eV}$ (IPS) and $4.19 \pm 0.08 \mathrm{eV}(\mathrm{CB}+n)$ with respect to the Fermi level, respectively. Only the data points with the four highest photon energies were used to determine the energy. Due to their dispersion behavior and shift with respect to the vacuum level, which are discussed below, we assign these states to the IPSs and to a higher-lying conduction band $(\mathrm{CB}+n)$ of the CGNRs.

Figure 3(b) shows the peak shift of the IPS and the CB $+n$ features, respectively, for varying $h v$. In both cases, the peaks associated with unoccupied states do not shift with an integer slope of 1, which would be expected in this case. Instead, there are different photon energy ranges in which the peak shift occurs with different slopes. Taking the four data points at the highest photon energies (where the two peaks are most pronounced) as a reference, the fitted slopes are $0.3 \pm 0.3$ (a)

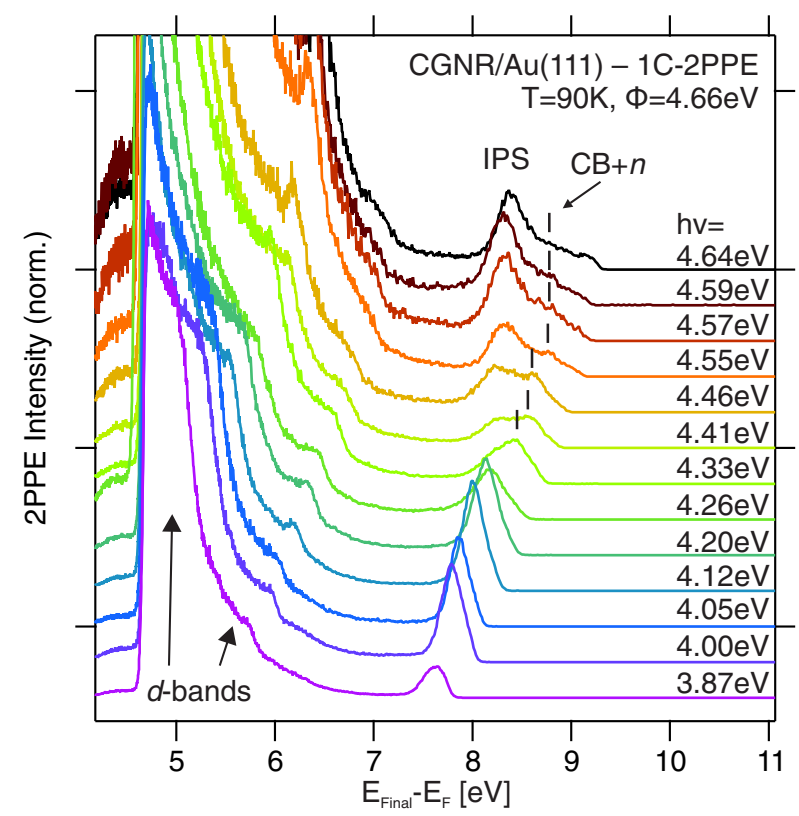

(b)

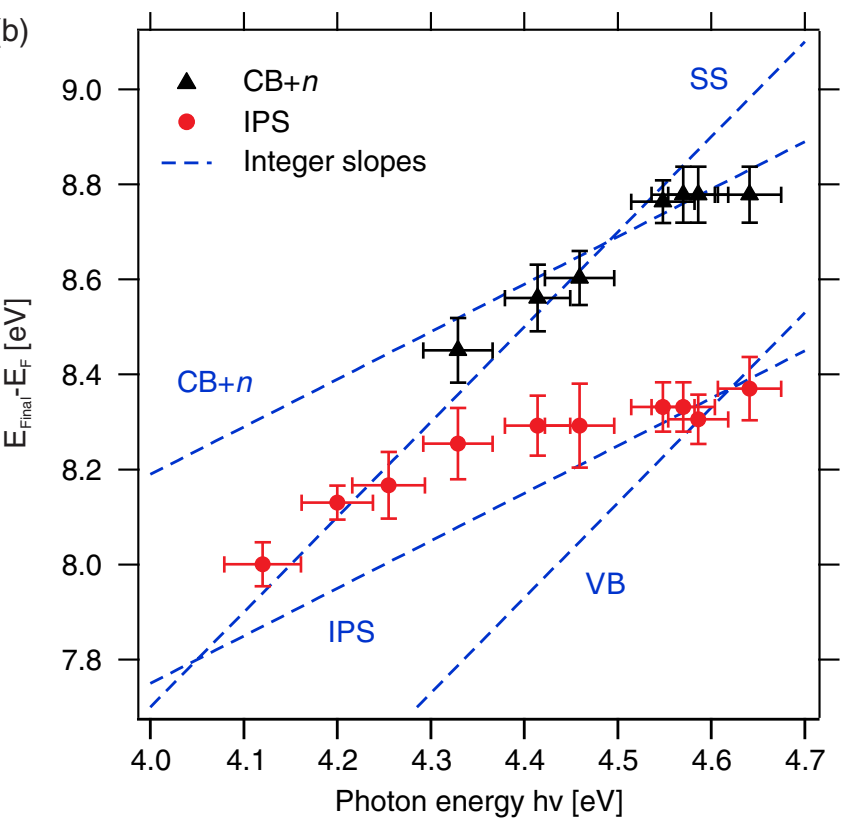

FIG. 3. (Color online) (a) 1C-2PPE spectra of the undoped CGNRs for different photon energies $h v$, recorded at $90 \mathrm{~K}$ sample temperature. Besides the gold $d$ bands, features from the IPSs and the $\mathrm{CB}+n$ are observed. The spectra were normalized with respect to one of the $d$-band features. (b) Shift of the final-state energy of the IPS and CB $+n$ features shown in (a) as a function of photon energy. The blue dashed lines represent the calculated final-state energy shift based on the measured energy of the occupied states (VB and SS) with $2 h v$ added [43] and the energy of the two unoccupied states as determined from the four high-energy data points.

and $0.1 \pm 0.8$ for the IPS and the $\mathrm{CB}+n$, respectively. In order to understand this behavior we included simulations of the final-state energies which peaks would have that arise from 2PPE from the two occupied states known for this system based on ultraviolet photoemission spectroscopy (UPS) experiments [43], namely the valence band (VB) at $-0.87 \mathrm{eV}$ and the surface state (SS) at around $-0.3 \mathrm{eV}$. The respective 
energy shifts are shown as a blue, dashed line with slope 2 in Fig. 3(b). These occupied electronic states cannot be observed in the present 2PPE spectra, presumably due to low transition dipole moments, compared to those of the unoccupied states. We also added calculated energy shifts for the two unoccupied states observed here but based only on the highest four photon energies at which the features are best separated (also blue, dashed lines, with slope 1). We ascribe the deviation of the actual peak positions from these energies to resonances with the occupied states, in particular the SS, which furthermore lead to an intensity increase of the 2PPE features. An excitation from occupied bulk states into the IPSs and the $\mathrm{CB}+n$ are another possibility, but the tendency of the two corresponding peaks to align with the simulated shift of the SS suggests that this is the occupied state from which the unoccupied ones are pumped.

The binding energy of the IPSs with respect to the vacuum level amounts to $0.91 \mathrm{eV}$. Despite the experimental error, this is an indication that it exceeds the general maximum binding energy of an $n=1$ IPS which is $0.85 \mathrm{eV}$. In order to understand this, one must distinguish between the global work function of the system and the local work function at the CGNRs which correspond to the respective vacuum levels. Since the CGNRs cover only approximately two-thirds of the sample surface [43], the global work function lies between the work function of clean $\mathrm{Au}(111)$ (see above) and the local work function in the vicinity of the GNR. The global work function is the quantity measured in 2PPE, which is a spatially averaging technique. In contrast, the IPS is pinned to the local vacuum level, which evidently is lower than that of the pristine surface.

In order to obtain more information about the two observed unoccupied states, we performed AR-2PPE, the results of which are shown in Fig. 4(a). Besides the $d$-band features which do not exhibit a dispersion parallel to the surface, we find that the $\mathrm{CB}+n$ feature appears localized, whereas the IPS shows a dispersive behavior. The fact that the GNRs are not aligned along a preferred direction [20] on the surface does not interfere with the measurement of the IPS since it is an isotropic, two-dimensional electronic state. We fitted the peak shift with a free-electron-like dispersion behavior according to

$$
E(k)=E_{0}+\frac{\hbar^{2} k^{2}}{2 m_{\mathrm{eff}}},
$$

which yields an effective mass of $0.63 \pm 0.04 m_{\mathrm{e}}$ [see Fig. 4(b)]. Note that we do not observe a peak associated with the conduction band of the CGNR which would be expected to disperse as well. As shown in the Supplemental Material [52], the singly (doubly) doped CGNR exhibit an analogous behavior with effective masses of $0.7 \pm 0.1 m_{\mathrm{e}}\left(0.8 \pm 0.1 m_{\mathrm{e}}\right)$. The effective mass thus increases with increasing dopant concentration. The reason for this behavior may be a less homogeneous potential energy landscape at the surface in case of the doped CGNR. The inhomogeneity due to the dopant atoms increases with increasing dopant concentration. Compared to the effective mass of $1.0 \pm 0.2 m_{\mathrm{e}}$ at the pristine surface $[28,29]$ and the mass of a free electron, the measured values are smaller, indicating more mobile electrons. This is a trend also observed for the IPSs of some (but not all [34,35]) adsorbed graphene systems [37]. (a)

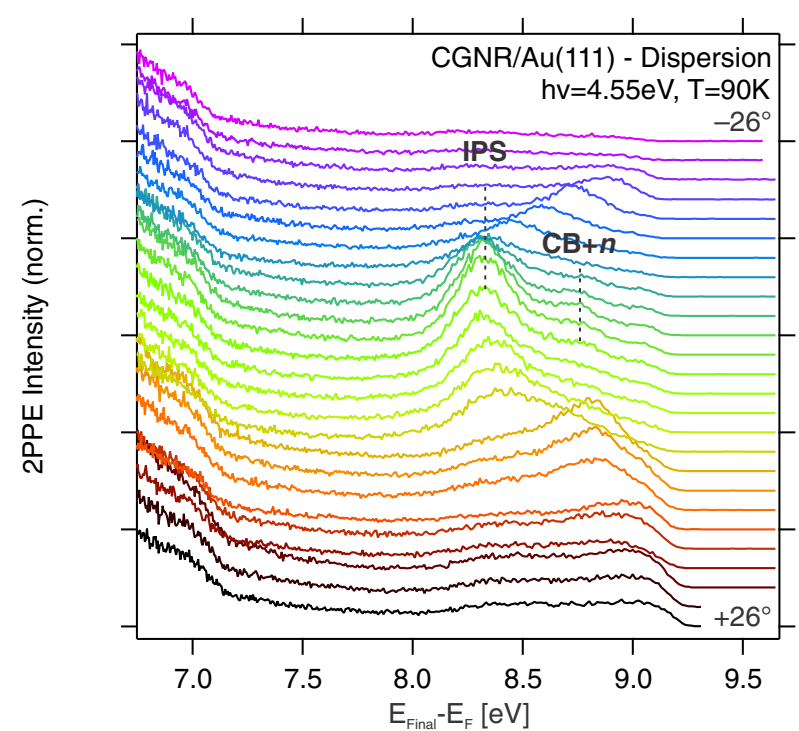

(b)

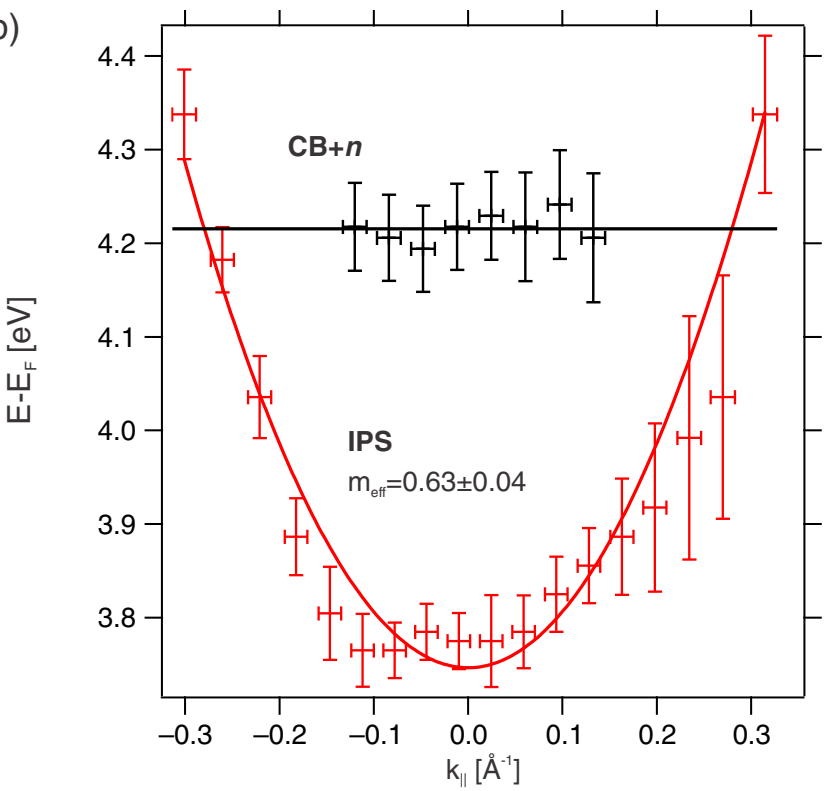

FIG. 4. (Color online) (a) 1C-2PPE spectra recorded for different emission angles. The spectra are normalized to one of the $d$-band features. The spectra give information about the dispersion behavior of the IPS and the CB $+n$ features. (b) Peak positions in the 2PPE spectrum as a function of the momentum parallel to the surface $k_{\|}$. The $\mathrm{CB}+n$ feature is shown along with the average energy and the IPS exhibits a free-electron-like dispersion behavior with an effective mass of $0.63 m_{\mathrm{e}}$. The energy given here is the binding energy of the IPSs with respect to the Fermi level, rather than the final-state energy of the photoelectron.

Time-resolved 2PPE allows us to investigate the population dynamics of the unoccupied electronic states, i.e., the IPS and the $\mathrm{CB}+n$. Figure 5 shows a two-color TR-2PPE measurement, meaning that the pump and the probe pulse have different photon energies $h v_{1} \neq h v_{2}$. In this measurement, the IPS and the CB $+n$ are pumped with the UV light of $h v_{2}$ and probed with the visible photons of $h v_{1}$. Therefore, the final-state energy of the photoemitted electrons is lower than in case of 1C-2PPE. In the false color plot of Fig. 5, this excitation scheme corresponds to the situation on the positive delay side, 


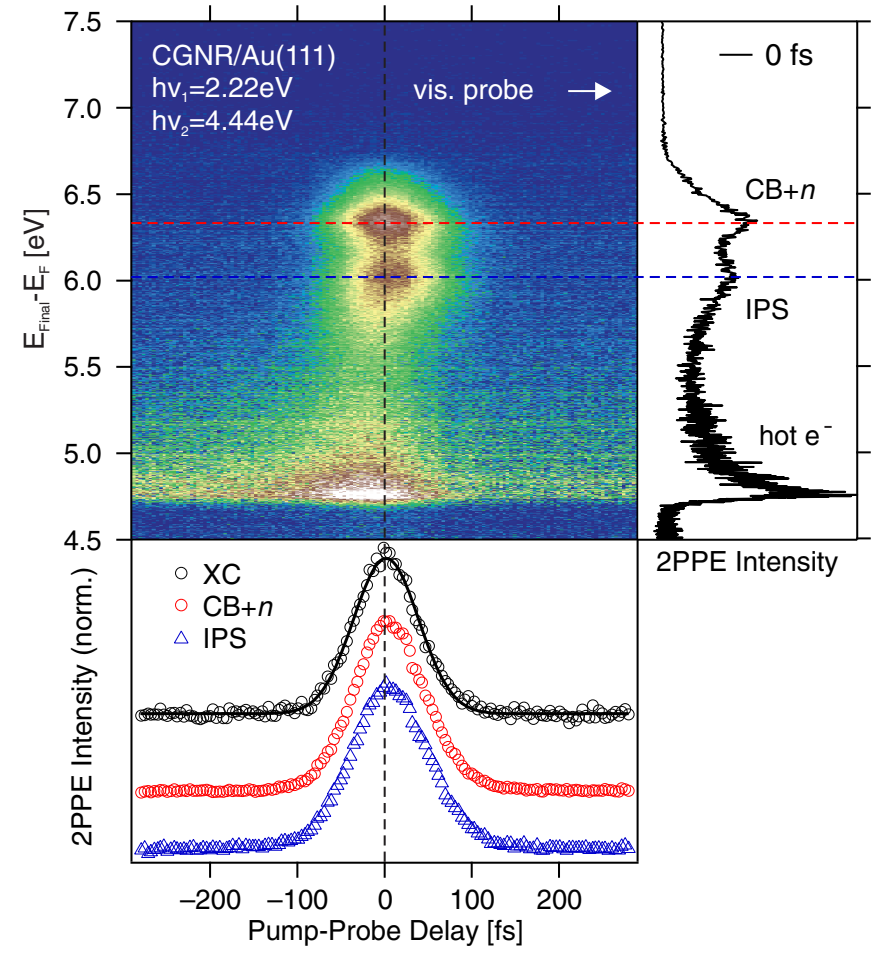

FIG. 5. (Color online) TR-2PPE recorded with laser pulses of different photon energies $h v_{1}$ and $h v_{2}$. The false color plot shows the 2PPE intensity as a color code (blue, low; white, high) as a function of the final-state energy and the pump-probe delay, where a positive delay value corresponds to the situation where the visible beam $\left(h v_{1}\right)$ acts as probe. In the bottom graph, the 2PPE intensity of the IPS and the $\mathrm{CB}+n$ features are shown along with the cross-correlation (XC) of the two laser pulses as measured in the energetic region around $6.7 \mathrm{eV}$. A fit of this XC curve yields the pulse width of an individual laser pulse, namely 62 fs (FWHM). The right graph shows a cut of the two-dimensional data set yielding a two-color 2PPE spectrum at vanishing delay.

meaning that a broadening of the cross-correlation trace would be observed toward that side. Besides the two features from the unoccupied states, a third feature is observable at final-state energies below $5 \mathrm{eV}$, which arises due to the excitation of hot electrons with the visible beam which are probed with the UV beam and thus display an asymmetry toward negative delay values.

The time resolution of the TR-2PPE measurement is essentially determined by the pulse duration which was measured by fitting a convolution of two Gaussian pulse profiles to the cross-correlation curve around the Fermi level, i.e., around a final-state energy of $6.7 \mathrm{eV}$. The resulting full width at half maximum (FWHM) of a single pulse is $62 \mathrm{fs}$. The corresponding cross-correlation trace is shown in the bottom part of Fig. 5 together with the cross correlations of the two features which are associated with the unoccupied states. For these two, no significant deviation from the cross correlation of the two laser pulses is observed, which means that the lifetime $\tau$ of the two states is below the time resolution. Also for the doped CGNRs, $\tau$ is not larger than 50 fs (see Supplemental Material [52]). The short lifetimes of the excited electronic states indicate effective de-excitation channels into the metallic substrate and are on the same order of magnitude as those

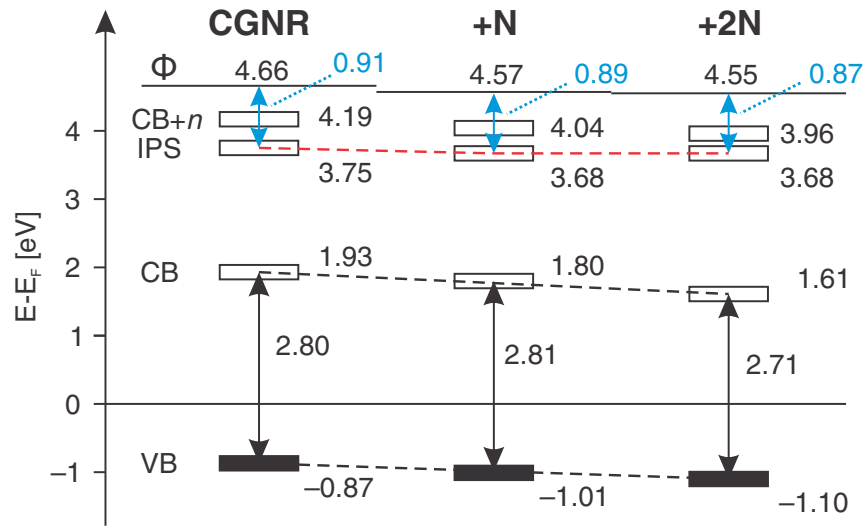

FIG. 6. (Color online) Comparison of the electronic structure of the pristine and the doped CGNRs with respect to the Fermi level. VB and CB values as well as the band gap were taken from Ref. [43]. See Supplemental Material [52] for details on the doped CGNRs.

measured for IPSs of adsorbed graphene systems [34,35,37], as well as an armchair GNR at the Au(788) surface [41].

Figure 6 shows a comparison of the results presented above for the pristine, undoped CGNRs with those for the doped CGNRs (see Supplemental Material [52]). The VB and CB values were taken from our previous work, Ref. [43], and were determined using photoemission and HREELS. There it was found that the increasing dopant concentration leads to a downshift of the CGNR band structure with respect to that of the underlying substrate, whereas the band gap remains nearly constant. The energetic position of the $\mathrm{CB}+n$ follows this trend of decreasing by roughly $0.1 \mathrm{eV}$ per $\mathrm{N}$ atom toward the Fermi level, as one would expect since the entire band structure is subject to this shift.

The binding energy of the IPSs, on the other hand, decreases slightly from the pristine to the singly doped CGNRs, but the energy does not further decrease upon addition of the second $\mathrm{N}$ dopant atom. This behavior can be understood by considering that of the vacuum level which follows the same trend. In fact, the binding energy of the IPSs with respect to the vacuum level remains nearly constant around $0.9 \mathrm{eV}$ for all three systems, as would be expected for an IPS. As already mentioned, the measured work function is higher than the local work function at the CGNRs, which is why the binding energy exceeds the expected maximum value of $0.85 \mathrm{eV}$.

\section{SUMMARY}

In summary, we have used 2PPE and its angle- and time-resolved variants to study the unoccupied electronic structure of a chevron-shaped CGNR on the Au(111) surface upon thermally induced on-surface synthesis. While we do not observe a signature of the $\mathrm{CB}$, we can observe the first IPS $(n=1)$ in the 2PPE spectra, as well as a higher-lying band of the CGNR. The peaks of these unoccupied states are subject to resonant excitation from the SS and the VB which are know from previous UPS measurements. While the $\mathrm{CB}+n$ appears localized, the IPS exhibits a free-electron-like dispersion with an effective mass of $0.63 m_{\mathrm{e}}$. Furthermore, we used TR-2PPE to study the population dynamics of the IPSs 
and find that the lifetime is below the time resolution of the experiment. Besides the pristine CGNRs we performed these experiments on two other systems with different $\mathrm{N}$ dopant concentrations. The results are similar except for an increasing effective mass and a downshift of the $\mathrm{CB}+n$ with respect to the Fermi level with increasing dopant concentration. Our study of the IPS at the CGNR/Au(111) interfaces adds to the research of IPS on graphene-derived nanostructures at metallic surfaces.

\section{ACKNOWLEDGMENTS}

We greatly appreciate synthesis of the precursor molecules by Marie Gille and Felix Brauße in the group of Stefan Hecht (Humboldt-Universität zu Berlin). We would like to acknowledge Celina Schulze, who contributed to the experimental work on the undoped CGNR in the framework of her Bachelor's thesis. Funding by the German Research Foundation (DFG) through collaborative research center SFB658 and Project No. TE479/3-1 are gratefully acknowledged.
[1] K. S. Novoselov, A. K. Geim, S. V. Morozov, D. Jiang, Y. Zhang, S. V. Dubonos, I. V. Grigorieva, and A. A. Firsov, Science 306, 666 (2004)

[2] K. S. Novoselov, A. K. Geim, S. V. Morozov, D. Jiang, M. I. Katsnelson, I. V. Grigorieva, S. V. Dubonos, and A. A. Firsov, Nature (London) 438, 197 (2005).

[3] A. K. Geim and K. S. Novoselov, Nat. Mater. 6, 183 (2007).

[4] Z. H. Ni, T. Yu, Y. H. Lu, Y. Y. Wang, Y. P. Feng, and Z. X. Shen, ACS Nano 2, 2301 (2008).

[5] T. M. G. Mohiuddin, A. Lombardo, R. R. Nair, A. Bonetti, G. Savini, R. Jalil, N. Bonini, D. M. Basko, C. Galiotis, N. Marzari, K. S. Novoselov, A. K. Geim, and A. C. Ferrari, Phys. Rev. B 79, 205433 (2009).

[6] F. Guinea, M. I. Katsnelson, and A. K. Geim, Nat. Phys. 6, 30 (2010).

[7] T. Ohta, A. Bostwick, T. Seyller, K. Horn, and E. Rotenberg, Science 313, 951 (2006).

[8] Y. Zhang, T.-T. Tang, C. Girit, Z. Hao, M. C. Martin, A. Zettl, M. F. Crommie, Y. R. Shen, and F. Wang, Nature (London) 459, 820 (2009)

[9] K. Nakada, M. Fujita, G. Dresselhaus, and M. S. Dresselhaus, Phys. Rev. B 54, 17954 (1996).

[10] M. Ezawa, Phys. Rev. B 73, 045432 (2006).

[11] M. Y. Han, B. Özyilmaz, Y. Zhang, and P. Kim, Phys. Rev. Lett. 98, 206805 (2007).

[12] Y.-W. Son, M. L. Cohen, and S. G. Louie, Phys. Rev. Lett. 97, 216803 (2006).

[13] X. Li, X. Wang, L. Zhang, S. Lee, and H. Dai, Science 319, 1229 (2008).

[14] F. Cervantes-Sodi, G. Csányi, S. Piscanec, and A. C. Ferrari, Phys. Rev. B 77, 165427 (2008).

[15] X. Wang, X. Li, L. Zhang, Y. Yoon, P. K. Weber, H. Wang, J. Guo, and H. Dai, Science 324, 768 (2009).

[16] H. Terrones, R. Lv, M. Terrones, and M. S. Dresselhaus, Rep. Prog. Phys. 75, 062501 (2012).

[17] Y.Zhang, Y.-W. Tan, H. L. Stormer, and P. Kim, Nature (London) 438, 201 (2005).

[18] D. V. Kosynkin, A. L. Higginbotham, A. Sinitskii, J. R. Lomeda, A. Dimiev, B. K. Price, and J. M. Tour, Nature (London) 458 , 872 (2009).

[19] L. Jiao, L. Zhang, X. Wang, G. Diankov, and H. Dai, Nature (London) 458, 877 (2009).

[20] J. Cai, P. Ruffieux, R. Jaafar, M. Bieri, T. Braun, S. Blankenburg, M. Muoth, A. P. Seitsonen, M. Saleh, X. Feng, K. Müllen, and R. Fasel, Nature (London) 466, 470 (2010).
[21] Y.-C. Chen, D. G. de Oteyza, Z. Pedramrazi, C. Chen, F. R. Fischer, and M. F. Crommie, ACS Nano 7, 6123 (2013).

[22] T. Fauster, C. Reuß, I. L. Shumay, and M. Weinelt, Chem. Phys. 251, 111 (2000).

[23] J. Güdde, W. Berthold, and U. Höfer, Chem. Rev. 106, 4261 (2006).

[24] A. Hotzel, Prog. Surf. Sci. 82, 336 (2007).

[25] M. Rohleder, K. Duncker, W. Berthold, J. Güdde, and U. Höfer, New J. Phys. 7, 103 (2005).

[26] C. D. Lindstrom, D. Quinn, and X.-Y. Zhu, J. Chem. Phys. 122 124714 (2005).

[27] K. Giesen, F. Hage, F. J. Himpsel, H. J. Riess, and W. Steinmann, Phys. Rev. Lett. 55, 300 (1985).

[28] D. Straub and F. J. Himpsel, Phys. Rev. B 33, 2256 (1986).

[29] D. P. Woodruff, W. A. Royer, and N. V. Smith, Phys. Rev. B 34 764 (1986).

[30] B. Borca, S. Barja, M. Garnica, D. Sánchez-Portal, V. M. Silkin, E. V. Chulkov, C. F. Hermanns, J. J. Hinarejos, A. L. Vázquez de Parga, A. Arnau, P. M. Echenique, and R. Miranda, Phys. Rev. Lett. 105, 036804 (2010).

[31] H. G. Zhang, H. Hu, Y. Pan, J. H. Mao, M. Gao, H. M. Guo, S. X. Du, T. Greber, and H.-J. Gao, J. Phys.: Condens. Matter 22, 302001 (2010).

[32] N. Armbrust, J. Güdde, P. Jakob, and U. Höfer, Phys. Rev. Lett. 108, 056801 (2012).

[33] D. Niesner, T. Fauster, J. I. Dadap, N. Zaki, K. R. Knox, P.-C Yeh, R. Bhandari, R. M. Osgood, M. Petrović, and M. Kralj, Phys. Rev. B 85, 081402(R) (2012).

[34] K. Takahashi, M. Imamura, I. Yamamoto, J. Azuma, and M. Kamada, Phys. Rev. B 89, 155303 (2014).

[35] A. J. Shearer, J. E. Johns, B. W. Caplins, D. E. Suich, M. C. Hersam, and C. B. Harris, Appl. Phys. Lett. 104, 231604 (2014).

[36] D. Nobis, M. Potenz, D. Niesner, and T. Fauster, Phys. Rev. B 88, 195435 (2013).

[37] D. Niesner and T. Fauster, J. Phys.: Condens. Matter 26, 393001 (2014).

[38] F. Craes, S. Runte, J. Klinkhammer, M. Kralj, T. Michely, and C. Busse, Phys. Rev. Lett. 111, 056804 (2013).

[39] M. Zamkov, N. Woody, S. Bing, H. S. Chakraborty, Z. Chang, U. Thumm, and P. Richard, Phys. Rev. Lett. 93, 156803 (2004).

[40] K. Schouteden, A. Volodin, D. A. Muzychenko, M. P. Chowdhury, A. Fonseca, J. B. Nagy, and C. V. Haesendonck, Nanotechnology 21, 485401 (2010).

[41] N. F. Kleimeier, A. Timmer, L. Bignardi, H. Mönig, X. L. Feng, K. Müllen, L. F. Chi, H. Fuchs, and H. Zacharias, Phys. Rev. B 90, 245408 (2014). 
[42] S. Linden, D. Zhong, A. Timmer, N. Aghdassi, J. H. Franke, H. Zhang, X. Feng, K. Müllen, H. Fuchs, L. Chi, and H. Zacharias, Phys. Rev. Lett. 108, 216801 (2012).

[43] C. Bronner, S. Stremlau, M. Gille, F. Brauße, A. Haase, S. Hecht, and P. Tegeder, Angew. Chem. Int. Ed. 52, 4422 (2013).

[44] C. Bronner, J. Björk, and P. Tegeder, J. Phys. Chem. C 119, 486 (2015).

[45] T. Fauster, Prog. Surf. Sci. 46, 177 (1994).

[46] H. Petek and S. Ogawa, Prog. Surf. Sci. 56, 239 (1997).

[47] M. Weinelt, J. Phys.: Condens. Matter 14, R1099 (2002).

[48] C. Bronner, G. Schulze, K. J. Franke, J. I. Pascual, and P. Tegeder, J. Phys.: Condens. Matter 23, 484005 (2011).
[49] C. Bronner, M. Schulze, S. Hagen, and P. Tegeder, New J. Phys. 14, 043023 (2012).

[50] P. Tegeder, J. Phys.: Condens. Matter 24, 394001 (2012).

[51] R. Paniago, R. Matzdorf, G. Meister, and A. Goldmann, Surf. Sci. 336, 113 (1995).

[52] See Supplemental Material at http://link.aps.org/supplemental/ 10.1103/PhysRevB.91.045428 for a detailed discussion of the corresponding experiments.

[53] C. Reuß, W. Wallauer, and T. Fauster, Surf. Rev. Lett. 03, 1547 (1996).

[54] S. Hagen, P. Kate, F. Leyssner, D. Nandi, M. Wolf, and P. Tegeder, J. Chem. Phys. 129, 164102 (2008).

[55] C. D. Lindstrom and X.-Y. Zhu, Chem. Rev. 106, 4281 (2006). 